\title{
Hula Hoop in Fitness and the Centripetal Force
}

\author{
Alicia Garcia-Falgueras \\ Spanish University, Madrid, Spain \\ Email: algarfal@gmail.com
}

How to cite this paper: Garcia-Falgueras, A. (2016). Hula Hoop in Fitness and the Centripetal Force. Psychology, 7, 1503-1517. http://dx.doi.org/10.4236/psych.2016.713147

Received: September 28, 2016

Accepted: November 5, 2016

Published: November 8, 2016

Copyright $\odot 2016$ by author and Scientific Research Publishing Inc. This work is licensed under the Creative Commons Attribution International License (CC BY 4.0)

http://creativecommons.org/licenses/by/4.0/

c) (i) Open Access

\begin{abstract}
Since centuries ago or even more, the hula hoop has been practiced by people to keep their good health while having fun. Curiously, this simple toy made by a plastic ring, also previously made by a circle of willow, rattan, grapevines or stiff grasses, is subjected to several high and complex physical laws that make, at the same time, possible its beautiful movements. Physical laws are opposing and/or accompanying the movement of the circle-hoop-ring, such as gravity, centripetal force, friction, speed or velocity, and acceleration, in a torque or tendency to rotate. Specific muscles of the body are activated during hula hoop dancing and a general cardio exercise is happening. On the other hand, a stimulation of creativity is also occurring to make a mathematically reasonable and beautiful flow of movements for the own enjoyment or to make a performance for other people's enjoyment. Then, the dancer with a hoop is moving the ring around several parts of his/her body (waist, core, shoulders, neck, arms), also rolling it over the body like a wheel, making waves in different axis, tossing it to the air or floor, etc. and dancer can jump him/herself through the hoop, or stealing its movements with his/her body. While motor learning happens, specific areas of the brain are activated differently during trials of learning. Then flow and fluid movements become automatic in an asymmetrical way considering our hand preference. However, for getting all this benefits, it is required a controlled practice in children and adults, that means a smooth and not violent, reasonable and measured practice in time and intensity and not negligent or irresponsible. Otherwise, some lesions have been described in literature.
\end{abstract}

\section{Keywords}

Centripetal Force, Hula Hoop, Gravity, Friction, Speed, Torque, Round Movements, Tosses, Jumps, Waves, Cerebellum, Cingulated Motor Area, Core, Abdominal Muscles, Pelvic Floor Muscles

\section{Introduction}

Hula hooping is a complex skill in which an unstable object, a hoop or a perfect ring in 
its circle shape, is kept in steady oscillation parallel with the ground or other planes by means of coordinate oscillations of different parts of the body (waist, hips, arms, legs, neck, knees, ankles, etc.), in uncountable variations of creativity or imaginative flows. Some specific exercises have been done for years, since the antiquity Greek or even earlier with the Egyptians. These exercises activate the cardio system in a funny way.

The activity of dancing a hula hoop is based on several physical laws, such as gravity, rotation, torque forces, centripetal forces, friction, inertia or eccentric movements. These physical laws are included in the game and outwitted via subtle application of impulses opposing the gravity and in the plane of the hoop (horizontally or vertically), producing changes in the angular movement of the hoop, till it reaches the momentum of being "orbiting" that has to be kept and maintained. In an experimental study, it was proved each hooper seems to apply his/her own strategies in maintaining hoop oscillation, despite equivalent experimental conditions occurred: 1) some hoopers used extensor movement of the knee to make the rotary motion, 2) other hoopers preferred a balanced strategy toward two directions moving two legs, waist and sides in equal proportion and 3) some other hoopers practiced the ankle-hip movement strategy in a more diagonal elliptical motion (Cluff et al., 2008).

Its shape is reminding also the number zero (0) or the letter vocal (O). Curiously, German and Latin numbers were letters ( $\mathrm{I}=$ one; $\mathrm{V}=$ five; $\mathrm{X}=$ ten; $\mathrm{L}=$ fifty; $\mathrm{C}=$ one hundred; $\mathrm{D}=$ five hundred and $\mathrm{M}=$ one thousand). In the $13^{\text {th }}$ century, zero was considered as something that "signifies nothing" but instead "holds a place and signifies for others". And the bigger the absence, the greater the potential presence. It is important to keep in mind algebra have an Arabic origin, (Al-Khwarizmi) that was incidentally modified as algorithm after the Latin transcription in $9^{\text {th }}$ century (Tammet, 2013).

\section{Physics}

From Math as the one exact Science, we would like to explore the inherent Physical theories in this game. Different movements of the body in its dance with the hula hoop might have the same kinetic and visually consequences (Balasubramaniam \& Turvey, 2004). However, it is important to keep in mind the ellipse path that for some movements is required, cannot be interfered nor finding any obstacle in its natural route, otherwise the movement is broken and the visually beauty of mathematically meaning or sense is destroyed. In an experimental study, a Fourier spectral analysis of frequency of the body movement of different persons while waist hoop oscillation and balancing revealed that his/her ankle, knee and hip oscillated at approximately the same frequency during movement. After an analysis over kernel Hilbert spaces algorithms while dancing movements, it was proved those body oscillations are faster and having smaller amplitude when the hoop is smaller than with a large hoop (Balasubramaniam \& Turvey, 2004).

One important concept to consider in this game is the centripetal force. It is described by Newton as "a force by which bodies are drawn or impelled, or in any way tend, towards a point as to a centre" (Figure 1). The mathematical description and its calculation were improved and derived in 1659 by the Dutch physicist Christiaan 


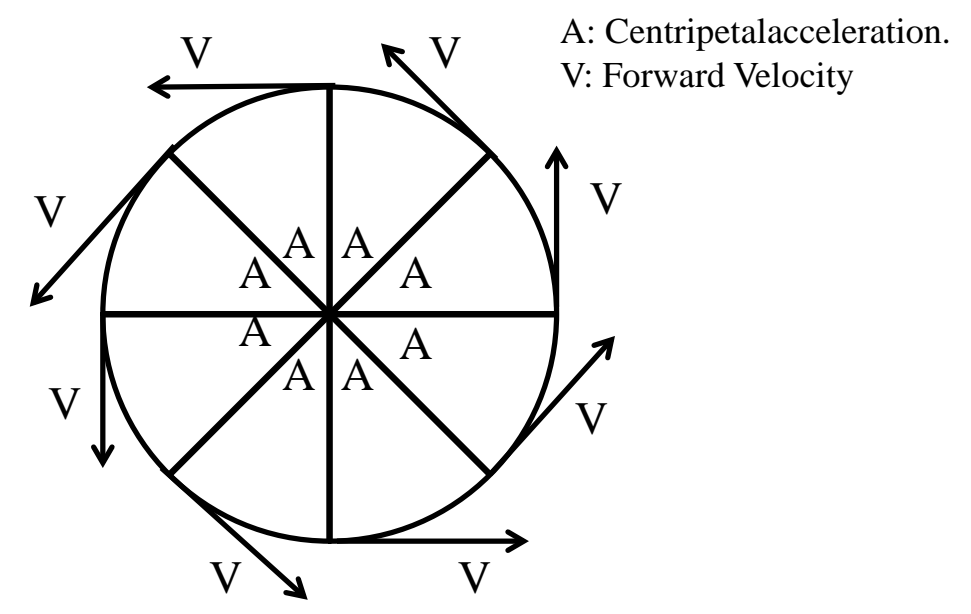

Figure 1. Schematic representation of a circumference in movement as a hodograph, counterclockwise direction: centripetal acceleration $(A)$ is the eccentric energy that goes outside the circle, while the velocity $(V)$ is the intensity of movement in a specific time $(\mathrm{mts} / \mathrm{sec})$. Those are the parameters to calculate the centripetal force $(F)$ : Mass of the body $(m)$ multiplied by the velocity squared $\left(v^{2}\right)$ and divided by the radius of circumference $(r): F=\left(m v^{2}\right) / r$.

Huygens. The magnitude of inward displacement along the axis aligned with centripetal force generally increases or decreases depending on the radius length for targets traveling an orbit with a constant angular velocity.

Looking at the stars, there is a coincidence, in scale, between the centripetal acceleration at which Newtonian gravity fails in equilibrated galaxies and the cosmic accelerations. In gravitationally bound systems from the length scale of dwarf galaxies up, the velocity of the objects inside the system exceeds the local escape velocity in the gravitational potential and that is how equilibrium happens (Starkman, 2011). Also looking at the smallest particles, even in crossed molecular beam experiments, these physical quantum dynamics events happens as long as no mixed chemical reaction occurs while the motion oscillation occurs. As in the hula hoop in both cases there are also arising forces from the interference between near-side and far-side scattering amplitudes (Wang et al., 2008) and the velocity inside the hoop has to exceeds the local escape velocity in gravitational potential to keep equilibrium.

For physical objects, Newton's first law of motion specifies that subsequent target motion should not differ from previous target motion in the absence of any other influences. This exercise of hula hooping is having some similarities to the planets movement around their orbits, studied by Newton and Kepler and visually represented with hodographs (Figure 1). Concerning to velocity, the Keplerian concepts of motion includes the shape of the object and its trajectory in velocity space (or momentum space). For each point on the spatial trajectory, it is possible to establish a formula with a corresponding velocity vector and its parallel direction to the tangent of the spatial trajectory at the point in question (Figure 1). The hodograph (name given by Hamilton in 1846) of the velocity vector, is constant for a circular orbit. The circular Keplerian 
motion the hodograph is itself a circle whose center is located at the origin of the velocity of the body at the vertex (torque) of the parabola is in the space. The radius of this circle equals the constant magnitude of circular velocity. Moreover, in other cases as a curious data, the circular form of the hodograph is characteristic not only of closed (circular and elliptical) orbits, but also of open (parabolic and hyperbolic) orbits of motion (Butikov, 2000).

Centripetal force is also having other uses in nature. For example, the movements that some fury mammals are doing when they want to dry their hair by rapidly oscillating their bodies in a similar process to shivering is based in centripetal forces. Some mammals such as lambs, are producing Lanolin that is a sterol esters with waterproofing properties and it helps sheep to shed water from their coats. There is an inverse correlation between fiber diameter and wool wax content. A study was done in five animals (squirrel, black bear, brown bear, lion and tiger) and the hodograph they got from their movements in waves are very similar to those obtained from hula hoop oscillations (Figure 2) (Dickerson et al., 2012). Through chest girth measurements and videos of the animals they concluded each animal has a particular frequency at which it shakes, depending on its size. They stop shaking to save energy when accumulated water is almost gone or when it is obtained a reasonable amount of dryness about the $70 \%$ saving energy. The larger animals in size have a bigger range of shaking of $4-6 \mathrm{~Hz}$,
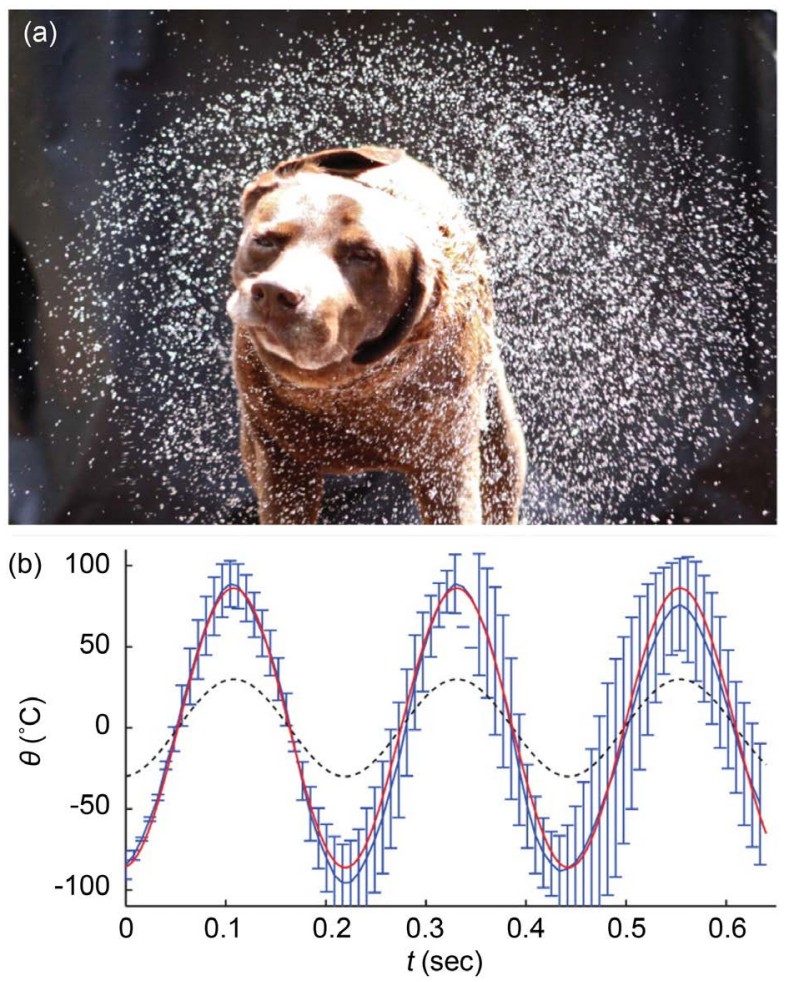

Figure 2. (a) A droplet cloud generated by a Labrador retriever during mid-shake; (b) Time course of the angular position of the skin and vertebrae of the $\operatorname{dog}(N=3)$. (Modified from Dickerson et al., 2012; with permission). 
(inside the best fit of 3.2 - $9 \mathrm{~Hz}$ ) (Dickerson et al., 2012). This procedure is aimed to pursuit a thermal restoration after cold water, because otherwise, the animals could lose dermal and thermal balance. The increase in amplitude of movement improves the efficacy of the shake through increasing the centrifugal force on drops within the fur. On the other hand, hairless mammals might have no shaking instinct.

As one of the main opposite forces to move a hula hoop, we find the gravity. It is a force that is very present during hula hooping, although curiously it is not included in the centripetal force formula, despite the fact it is having an effect on acceleration via velocity. The gravity, that is a sort of "vertical velocity" of $9.8 \mathrm{mts} / \mathrm{sc}$ in Earth, is influencing the minimum impulse to start and keep the hula hoop in its dancing. The size of the hula hoop is having an effect because of gravity. Actually, in space for astronauts it is not possible to play any hula hoop at all, because of the total lack of gravity but also because of the total lack of references for orientation, scaling and calibration of time and space (Lacquaniti et al., 2015). Not in vain, for beginners in hula hoop dancing the main frustration is the incapacity for controlling gravity over their exercises, because hoop drops down so many times while practicing.

Another important concept to consider to twirl a hula hoop is the movement around the waist or along one axis (torque). It was calculated this movement has a phase difference lying between $\pi / 2$ and $\pi$ (Seyranian \& Belyakov, 2011). A torque, moment, or moment force is the tendency of a force to rotate an object about an axis or pivot. It is represented as the Greek letter tau and its magnitude depends on three qualities: the force, the ratio or lever arm connected to the axis and the angle between the force vector and the lever arm. That is why variables such as the radius of the hula hoop $(R)$, the radio of the waist hooper or torque $(r)$, are having an effect on the oscillation and its amplitude or other parameters (Figure 3).

However, for hula hoop movements an accurate mathematical formula is not possible to provide, since the movement is facing with many obstacles and interruptions that do not occur for planets orbits, for instance having an "emotional torque" (that is the person). When the person inside the hula hoop is moving his/her body, it can be done as spin movement (to propel the hoop around) or as a robbed movement (following the natural movement of the hula hoop with his/her body for visually flowing). Then, the dancer with a hoop is moving the ring around several parts of the body (waist, core, shoulders, neck, arms), also rolling it over the body like a wheel, making waves in different axis, tossing it to the air or floor, etc. and he/she can jump him/herself through the hoop, or to steal its movements with his/her body. Endless creativity and variations cannot be mathematically calculated.

Moreover, the friction is a component of the science of tribology that happens when two surfaces in contact move relative to each other and it converts kinetic energy into thermal energy (as fired might be started with wood friction). That is the reason why dancing with a cold hula hoop over skin is annoying but quickly the thermal sensation is equaled. That variable of friction is not included either in centripetal force formula, although it might have an effect over velocity. Then a simplified schema for this 


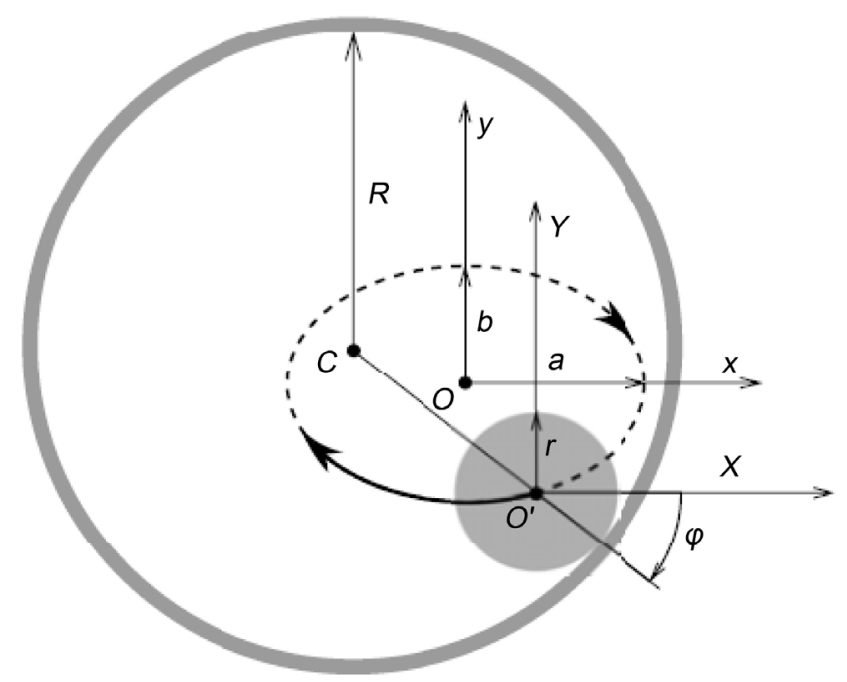

Figure 3. In this figure, center of the big hoop $(O)$, the torque $(O)$ and their different parameters are included as different variables to consider. The big radius $R$ is twirling with the angle $\varphi$ around a circular waist (torque-person shaded) having inside the smaller radious $r$, that might also be considered as the torque (tau). The center $O^{\prime}$ of the waist moves along the elliptic curve with the fixed center $O$. (From Seyranian \& Belyakov, 2011 with permission).

movement and forces against could be exposed as following:

Centripetal force might be happening outside the smaller radius $(r)$ by the torque ( tau) (person) who is controlling the centripetal acceleration by the forward velocity of his/her drive in oscillation. That is the reason why, when the hooper is dancing with the hoop in his/her both legs together, have a forward velocity $(z)$ that changes into a slower one $(z)$ when the oscillation is suddenly changed from two to only one leg, because the radius of one leg is smaller than two legs together, so do the forward velocity reducing the centripetal acceleration of movement.

Concerning to the "human torque", we will explain some muscles involved. The simple fact of walking is requiring the equilibrium that the central nervous system organizes through the movement of propulsion connected to body rotations. Turning during walking is a complex activity of the head, the trunk, the pelvis and feet reorientated together with adjustments of the body and counteracting the centrifugal acceleration (Turcato et al., 2015). Apparently, the optimization of movements for the better effect in action is a selection of muscle activation based on principle minimization of muscle recruitment, precision and speed of neural calculation. However, this constant balance of activity and movement is required to be adapted to each different movement and activities of the any movement including hoop dancing. The professional body builder Pauline Nordin wrote "Going all out and then trying to maintain that tempo in your life. You cannot be a sprinter and think you are going to be able to keep that speed for a marathon" (Pauline Nordin).

However, as a fitness activity, hula hooping might be funnier than walking: in a ex- 
perimental study it was proved by comparing the practicing of fitness via walking and via hula hooping in different people, that the participants who practice hula hoop were more positively activated affected and had stronger intentions for future aerobic exercise compared to those participants assigned to walk (Stevens et al., 2016).

Hula hooping is a sport that requires body skills and balance as many other sportive activities. It is a sport, not only because it increases body metabolism and tone belly, butt and thighs burning about 200 calories in 20 - 30 minutes (Zamor, 2009) or 600 calories in one hour, being higher energy consuming than an advance Pilates class. It is also a sport because it provides clues for improving posture, proprioception and balance, that is the manner we move our bodies in the world (Garcia-Falgueras, 2016).

But hula hoop is also a sport/game because it includes the second, third and fourth characteristics of sports or games definition in Homo Ludens (by Huiztinga, 1944) and by Salen and Zimmerman in 2006, "The Game Design Readers", that are Ilnex, Mimicry and Alea but not Agon. Specifically Ilnex is the compound of producing in oneself, by a rapid whirling or falling movement, a state of dizziness and disorder. That might be certainly one of the funniest effects of hula hooping, after rotation the body together with the hoop, in spinning movement, a slightly dizziness happens and makes the hooper to smile having fun, in much better way that taking any drug or unhealthy substance. More intense Ilnex has been also described in risks sports such as bungee jumping or rafting, but those involve speed, height a high level of physical exertion and highly specialized gear. On the other hand Mimicry is the aspect of becoming a different person while the game occurs, as many hoopers do with beautiful and imaginative costumes, fancy dresses and characterizations to create and to be a specific hooper. Alea is happening because of the compound of randomness and unpredictable behavior of natural sources, that makes the hula hoop falling down or changing its direction in unexpected ways, as many match points in sports. The other component of games, such as Agon, (the competitive side of games) does not exist in this game-sport of hula hooping, because competition is made at individual level and there is no score or punctuation rules for this practice.

Nowadays, the main way of learning how to create and elaborate choreographies of dance, to refresh new movements, to learn new tricks, variations or different styles of hooping is through internet with youtube, giving another new and revolutionary network dimension to the Mimicry aspect of this game. There are thousands of tutorial videos, online hula hoop trainers such as those made by Betty Lucas, Natalie "McFancy" Wise, Katie Sunshine or Deanne Love and many other spontaneous isolated examples of normal people, girls and boys, who are not athletes nor gymnastic, but who are dancing and having fun in their own style. There are many web pages specialized in this game (www.hooping.org) where people are continually posting and showing generously their imaginative and new beautiful flows, in different landscapes and frames all around the world and offering almost infinitive variables and stimulant ways to play this simple toy. The enormous variety of tutorials about how to make motor magic with a simple plastic ring are of good use for motivation of people who wants to learn or 
have different founts of inspiration from different people and cultures. People are sharing this funny and healthy entertainment via youtube since 2003, as far as we know.

\section{Muscles}

Several muscles are involved in this exercise, according to its huge variety and creativity. We would say, the main muscles that are usually activated are those belonging to the core, the Power House or the electric center of the body, as Pilates named them (Pilates, 1945). Curiously they are in the center of the body, equidistant to arms and legs, as the center of the hoop. These might be the basic set of muscles involved in basic hula hoop dancing, that are the abdominal muscles, internal and external oblique, transversus $a b$ dominis, but also muscle from the pelvic floor such as piriformis and coccygeus might be involved (Figure 4).

Another important muscles activated in basic movements are those belonging to the back (dorsal) and gluteus. They are also very important to keep body balance together with abdominals in the "go and see" movements. In eccentric, divergent, outward and efferent movements radiating from a central point of the body and hoop, considering the ab/adduction axis, these other following muscles might algo be activated: in the shoulders muscles (deltoids), in arms (biceps, triceps) and legs (cuadriceps and femorals) and infloxing flexor and extensor muscles of the ankle, knee and hip joing, being different for each person and flow. The more complex the dance, the bigger amount of muscles involved and the more complete the exercise with flexibility and almost imposible postures as an extreme.

\section{Neuroscience of Automatic Movements}

Muscles are activated from the brain. While the movements of the hula hoop are being learned, attention, monitoring or working motor memory are very required to find the correct set of muscles and movements to activate for getting the desired effect,
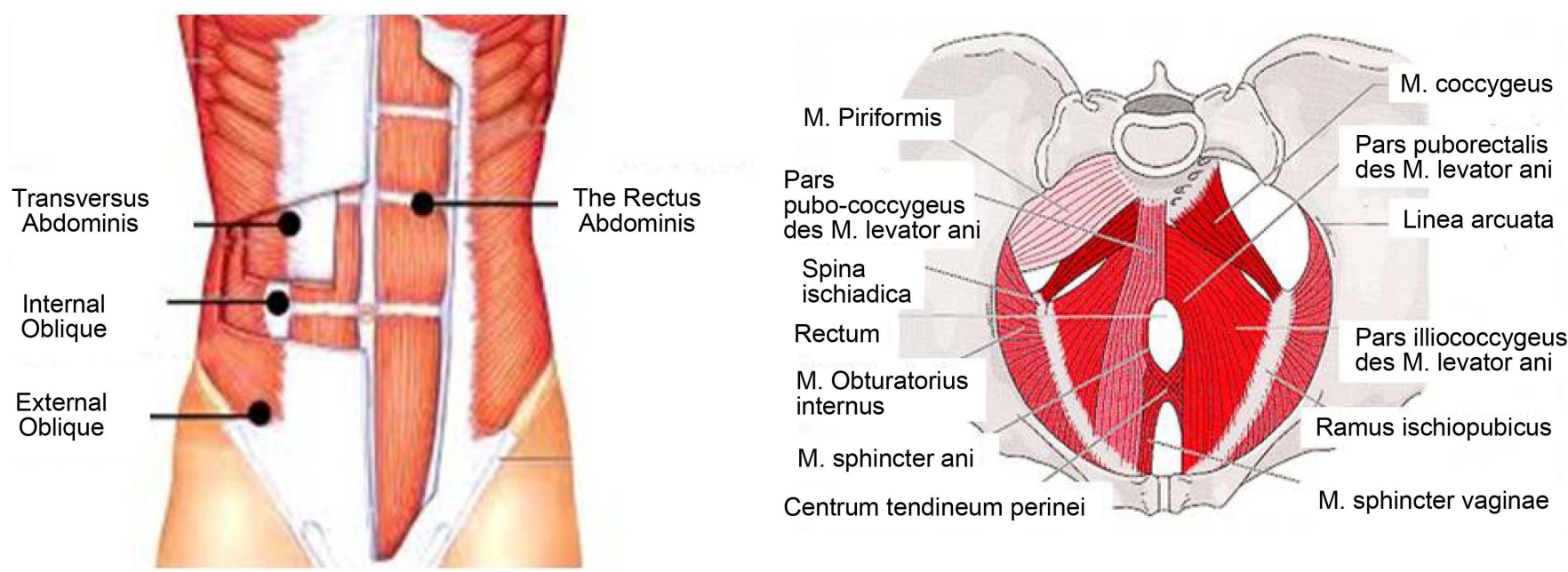

Figure 4. Muscles in the abdomen (left) and pelvic floor (right) that might be activated during basic hula hoop movements, to keep body balance through variation and oscillations of dancing. 
considering the physical laws as well. Once the movement has been automated, those attentional, monitoring or working memory areas in the brain are less activated and another specific motor networks become relevant.

In a psychological basic experiment it was found of our mental representation of motion is a priori existing in our brains. The manner we perceived and memorized movements in circles to predict the position of a target in movement traveling along a circular orbit, is depending on this a priori representation, as it was proved by Freyd and Jones (1994). They found that the largest magnitude of representational momentum along the spiral straingh-line path, the smallest accurate representation momentum occurred and, subsequently, the bigger probability to predict wrongly (Hubbard, 1995). That is, our a prioristic representation is failing when the time is very long. The a priori representation of centripetal force in our brains might be providing a cognition or conceptual representation about centripetal movements that might affect our predictions depending upon expectations concerning future target behavior (Hubbard, 1993, 1995).

In an experimental study, a sequential movement of the right finger with a specific sequence was studied and its representation in the brain. They found when the subject was able to repeat the movements 10 times in a row without errors, the automatization might have occurred. The areas more activated at automatic stage were the cerebellum (C3, zone in lobes IV and V; Dean et al., 2013), cingulate motor area (CMA), supplementary motor areas (M1) and putamen, whereas the precuneus nucleus (attention networks) in the brain had less connectivity. This study gave a new meaning to the concept of functional and effective connectivity (Wu et al., 2008) (Figure 5).

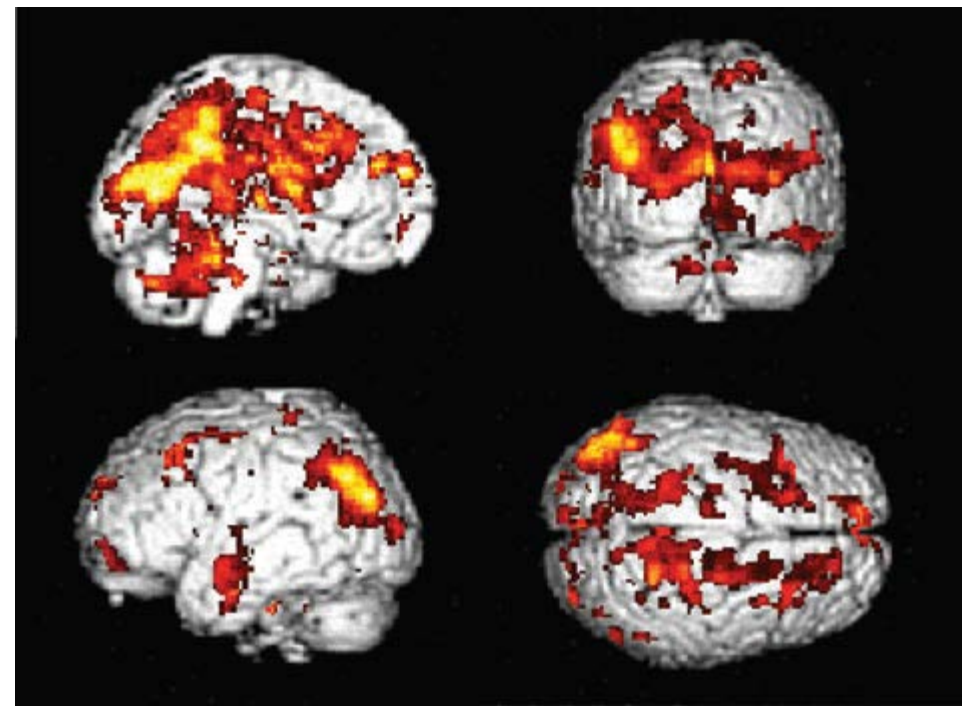

Figure 5. MRI pictures showing different brain areas significantly more activated while finger sequence motor learning activity as becoming automatic compared with the novel stage $(p<0.05$ corrected for multiple comparison). Top two figures show the cerebellum and cingulated motor areas (CMA) activated and two down pictures shows structures in motor cortex, M1. Prefrontal cortex is not very active in this picture (From Wu et al., 2008 with permission). 
For an automatization of movements to become more efficient and shifted more subcortically, the brain functions is dual: both activation from motor (primary motor cortex, M1) and from cognitive (cingulate motor area, CMA) have to happen (Wu et al., 2004, 2008) together with some cerebellum structures as well (C3 region, its molecular layers with interneurons and the parallel fibre synapses on the Purkinje cells; Dean et al., 2013). Those cerebellum areas and cells have been hypothesized to be critical for the computation of the motor error signals necessary for implicit adaptation (Taylor et al., 2010). In the experiment mentioned, during the learning of movements self-initiated and memorized, there was no visual feedback to provide information for subjects whether their movements of presses were correct or incorrect (Wu et al., 2004), then proprioception areas of the brain might have been also activated. That is why isolation of one brain area over the other ones during specific exercises and motor learning is very complicated (Wu et al., 2004). A set of multiple brain areas, as well as multiple muscles, are activated per each movement, to provide efficiently and propioception during exercise (Taylor et al., 2010; Garcia-Falgueras, 2016).

Another clinical studies have shown that patients with some cerebellar pathology exhibit impairments in sensorimotor adaptations tasks, with persistent errors, that is not improved after practice. In these cases their performances remained relatively stable over the course of training with no improvements, depending on each degree of lession and adaptation in the patient (Taylor et al., 2010).

During hula hooping practice, the way of motor learning happens is via repetitions and repetitions again and again, although the right movement could suddenly happens by mere fortuity. During repetitions and adjustments at some point the right combination of muscles and forces get into a balance and a motor sequence is built with a fluid flow. When that happens, it is possible that the sequence just built would be activating another rewarding area in the brain, and then the learning process has occurred, keeping in memory for further occasions the same muscles, calculations and combinations. That fact might be deduced by the smile that occurs in hoopers when they success making a difficult performance. The sequence achieved could be further repeated and improved in details, polishing, removing gross movements till it is completely flow and automatic and the performance is visually impeccable and automatic.

On the other hand and because of the unexpected effects of natural forces (gravity, air, centripetal and other forces, Alea components), spontaneous surprising movements might suddenly occur during dancing and without preparation. The accurate and flow performance of those spontaneous movement will depend on $i$ ) the multiple sequences already printed for those or similar movements, ii) the brain calculations in milliseconds for the best combination of moves based on the previous experience and iii) the automatisms of dancing in consent harmony together with the exterior physical laws or forces, like "go with the flow".

\section{Symmetry}

Something of relevance that happens in common in the brain and in the muscles is the 
asymmetrical activation. Specifically for hula hoop practicing is also the asymmetric preference in motor learning. Because our brain presents motor asymmetry and laterality, for specific movements we prefer or feel more comfortable with one hand or feet rather than the other one. During hula hooping, as well as for some motor movements, the fluent and easily movements are done in one direction, i.e. counterclockwise for right-handed, but it fails and do not success in the opposite direction or with the other side of the body, i.e. clockwise for right-handed, being in both cases the same physical laws involved and having the motor sequence skill already been learned.

In an experimental basic study a direct network between human cerebellum and limbic system was beautifully measured and right-left symmetry was compared using probabilistic Constrained Spherical Deconvolution (CDS) tractography (Arrigo et al., 2014). Some authors have already described a number of asymmetric tracks of the uncinate fascicule, anterior thalamic pathway and fornices. A fractional anisotropy (FA) right-left intra study revealed significantly right-left variabilities (asymmetries) for the same track in the cerebellum-limbic pathway connections. This might be due because of interhemispheric differences regarding brain functions, axon diameters, number of cells as well fiber myelination, instead of microscopic white matter differences, for the same track in right-left hemispheres (Arrigo et al., 2014).

\section{Health}

As any matter of balance between forces, brain and muscles, some lesions, hazards and injuries might occur during hula hooping. Some of them have been clinically described in Scientific literature mainly because of a negligent or irresponsible use of the hula hoop or because previous diseases existed. Those were related to muscle inflammation (traumatic myositic), bruises (interstitial and muscular oedema), muscular pain (shoelace or stiffness), mild abdominal pain or redness of the skin (erythematous or scratches), allergies to plastic (urticarial wheals with itchings, dematitis), dizziness and headaches (Zaidi, 1959).

A clinical case was described in a woman of 63 years old who presented an acute back pain and sciatica after playing vigorous hula hoop during 8 hours (Yang \& Chang, 2003). After surgical decompression and evacuation of the spinal subdural hematoma by irrigation and aspiration, the patient recovered optimally with no residual hematoma nor postoperative neurologic deficits. It is generally agreed by most authors regard spinal subdural hematoma that evacuation with laminectomy should be performed as surgical emergency before the spinal cord is irreversibly damaged (Yang \& Chang, 2003). Another case of violent playing hula hooping leaded into a perirenal hematoma having the patient previously a clinical diagnose of diabetes mellitus that was treated with insulin (Park et al., 2007). This patient was not obese, but she did hula-hoop excessively every day in attempt to lose weight, then with repeating negligent practice everyday she added minor injury each time to her already injured kidneys because of diabetes, till a big unilateral hematoma (because of asymmetry) was made that required surgical intervention (Park et al., 2007). 
We suggest as a preventive consideration that another clinical cases where hula hooping or any other balance sports, might be practice very carefully, or even not practice at all, would be: 1) in persons who present very pronounce body and brain asymmetries (such as vestibular locomotor ataxias), 2) persons who have structural asymmetries such as idiopathic scoliosis (Byl et al., 1997), 3) cases of hemiparesis with no compensatory mechanisms (Balasubramanian et al., 2007) or 4) those woman who had tears in pelvic floor after traumatic dysfunctional partum (Chaliha, 2009).

\section{Conclusion}

Practicing this sport or fitness activity might have several health benefits, although some clinical facts have to be considered as well. Contrary to other games such as computers competitions, video games, and game console, hula hooping has to be done in the open air, in a spacious area and it is based on natural laws and principles of nature for working and having fun. Then at the same time the person is moving his/her body, oxygenating and expelling toxins, he/she might also be learning about complex laws that rule the world and learning new movements for next stages. Ilnux, Mimicry and Alea compound of games is present in hooping but its risk, under safe non violent or negligent conditions, might be very little or absent. A very interesting brain activation pattern happens during motor learning, since it implies motor learning and automaticity, although no experimental studies exist about the specific brain activation during hula hooping. Motor studies have been done comparing different levels of the body while dancing the hula hoop (knees, waist, neck...) and they reveal while one level of the body is carrying the main role with the oscillating hula hoop, the other parts of the body are equivalently moving in similar waves like a unique water container or different planes in a mirror.

\section{Acknowledgements}

I thank Prof. Dr. D. F. Swaab for his teaching and orientation in the method of studying and researching and to Dr. Jenneke Kruisbrink for her literature resource help.

\section{Funding}

This research received no specific grant from any funding agency in the public, commercial or not-for-profit sectors.

\section{References}

Arrigo, A., Mormina, E., Anastasi, G. P., Gaeta, M., Calamuneri, A., Quartarone, A., De Salvo, S., Bruschetta, D., Rizzo, G., Trimarchi, F., \& Milardi, D. (2014). Constrained Spherical Deconvolution Analysis of the Limbic Network in Human, with Emphasis on a Direct Cerebello-Limbic Pathway. Frontiers in Human Neuroscience, 8, 987.

https://www.ncbi.nlm.nih.gov/pubmed/25538606

http://dx.doi.org/10.3389/fnhum.2014.00987

Balasubramaniam, R., \& Turvey, M. T. (2004). Coordination Modes in the Multisegmental Dynamics of Hula Hooping. Biological Cybernetics, 90, 176-190. 
https://www.ncbi.nlm.nih.gov/pubmed/15052481

http://dx.doi.org/10.1007/s00422-003-0460-4

Balasubramanian, C. K., Bowden, M. G., Neptune, R. R., \& Kautz, S. A. (2007). Relationship between Step Length Asymmetry and Walking Performance in Subjects with Chronic Hemiparesis. Archives of Physical Medicine and Rehabilitation, 88, 43-49.

https://www.ncbi.nlm.nih.gov/pubmed/17207674

http://dx.doi.org/10.1016/j.apmr.2006.10.004

Butikov, E. I. (2000). The Velocity Hodograph for an Arbitrary Keplerian Motion. European Journal of Physics, 21, 1-10. http://butikov.faculty.ifmo.ru/Hodograph.pdf http://dx.doi.org/10.1088/0143-0807/21/4/303

Byl, N. N., Holland, S., Jurek, A., \& Hu, S. S. (1997). Postural Imbalance and Vibratory Sensitivity in Patients with Idiopathic Scoliosis: Implications for Treatment. Journal of Orthopaedic and Sports Physical Therapy, 26, 60-68. https://www.ncbi.nlm.nih.gov/pubmed/9243403 http://dx.doi.org/10.2519/jospt.1997.26.2.60

Chaliha, C. (2009). Postpartum Pelvic Floor Trauma. Current Opinion in Obstetrics and Gynecology, 21, 474-479. https://www.ncbi.nlm.nih.gov/pubmed/19855276 http://dx.doi.org/10.1097/GCO.0b013e328332a84e

Cluff, T., Robertson, D. G., \& Balasubramaniam, R. (2008). Kinetics of Hula Hooping: An Inverse Dynamics Analysis. Human Movement Science, 27, 622-635.

https://www.ncbi.nlm.nih.gov/pubmed/18405990 http://dx.doi.org/10.1016/j.humov.2008.02.018

Dean, P., Anderson, S., Porrill, J., \& Jörntell, H. (2013). An Adaptive Filter Model of Cerebellar Zone C3 as a Basis for Safe Limb Control? Journal of Physiology, 591, 5459-5474. https://www.ncbi.nlm.nih.gov/pubmed/23836690

Dickerson, A. K., Mills, Z. G., \& Hu, D. L. (2012). Wet Mammals Shake at Tuned Frequencies to Dry. Journal of the Royal Society Interface, 9, 3208-3218.

https://www.ncbi.nlm.nih.gov/pubmed/22904256 http://dx.doi.org/10.1098/rsif.2012.0429

Freyd, J. J., \& Jones, K. T. (1994). Representational Momentum for a Spiral Path. Journal of $E_{X^{-}}$ perimental Psychology: Learning, Memory, and Cognition, 20, 968-976. https://www.ncbi.nlm.nih.gov/pubmed/15052481 http://dx.doi.org/10.1037/0278-7393.20.4.968

Garcia-Falgueras, A. (2016). An Introduction to Proprioception Concept in Pilates and Yoga. British Journal of Medicine and Medical Research, 15, 1-3.

http://www.sciencedomain.org/abstract/14259 http://dx.doi.org/10.9734/BJMMR/2016/25540

Hubbard, T. L. (1993). The Effect of Context on Visual Representational Momentum. Memory and Cognition, 21, 103-114. http://link.springer.com/article/10.3758/BF03211169 http://dx.doi.org/10.3758/BF03211169

Hubbard, T. L. (1995). Environmental Invariants in the Representation of Motion: Implied Dynamics and Representational Momentum, Gravity, Friction, and Centripetal Force. Psychonomic Bulletin \& Review, 2, 322-338. https://www.ncbi.nlm.nih.gov/pubmed/24203714 http://dx.doi.org/10.3758/BF03210971

Huiztinga, J. (1949). Homo Ludens: A Study of the Play-Element in Culture. London: Routledge and Kegan Paul Ed.

http://art.yale.edu/file_columns/0000/1474/homo_ludens_johan_huizinga_routledge_1949_.pdf

Lacquaniti, F., Bosco, G., Gravano, S., Indovina, I., La Scaleia, B., Maffei, V., \& Zago, M. (2015). 
Gravity in the Brain as a Reference for Space and Time Perception. Multisensory Research, 28, 397-426. https://www.ncbi.nlm.nih.gov/pubmed/26595949

http://dx.doi.org/10.1163/22134808-00002471

Park, S. K., Kim, H., Lhee, H. Y., \& Lee, K. B. (2007). Development of a Perirenal Hematoma after Hula-Hooping. Yonsei Medical Journal, 48, 868-870.

https://www.ncbi.nlm.nih.gov/pubmed/17963347

http://dx.doi.org/10.3349/ymj.2007.48.5.868

Pilates, J. (1945). Pilates's Return to Life through Contrology. Presentation Dynamics (NY) Editorial. https://babel.hathitrust.org/cgi/pt?id=mdp.39015038069475;view=1up;seq=9

Salen, K., \& Zimmerman, E. (2006). The Game Design Reader. A Rules of Play Anthrology. Massachusetts: The MIT Press. http://nideffer.net/classes/270-08/week_01_intro/Caillois.pdf

Seyranian, A. P., \& Belyakov, A. O. (2011). How to Twirl a Hula Hoop. American Journal of Physics, 79, 712-715. https://arxiv.org/ftp/arxiv/papers/1101/1101.0072.pdf http://dx.doi.org/10.1119/1.3576177

Starkman, G. D. (2011). Modifying Gravity: You Cannot Always Get What You Want. Philosophical Transactions of The Royal Society A Mathematical Physical and Engineering Sciences, 369, 5018-5041. https://www.ncbi.nlm.nih.gov/pubmed/22084291

http://dx.doi.org/10.1098/rsta.2011.0292

Stevens, C. J., Smith, J. E., \& Bryan, A. D. (2016). Apilot Studyof Women's Affective Responses to Common and Uncommon Forms of Aerobic Exercise. Psychology \& Health, 31, 239-257. https://www.ncbi.nlm.nih.gov/pubmed/26394246 http://dx.doi.org/10.1080/08870446.2015.1095917

Tammet, D. (2013). Thinking in Numbers. On Life, Love, Meaning and Math. New York: Little, Brown and Company.

https://www.amazon.es/Thinking-Numbers-Maths-Illuminates-Lives/dp/1444737449

Taylor, J. A., Klemfuss, N. M., \& Ivry, R. B. (2010). An Explicit Strategy Prevails When the Cerebellum Fails to Compute Movement Errors. Cerebellum, 9, 580-586.

https://www.ncbi.nlm.nih.gov/pubmed/20697860

http://dx.doi.org/10.1007/s12311-010-0201-x

Turcato, A. M., Godi, M., Giordano, A., Schieppati, M., \& Nardone, A. (2015). The Generation of Centripetal Force When Walking in a Circle: Insight from the Distribution of Ground Reaction Forces Recorded by Plantar Insoles. Journal of NeuroEngineering and Rehabilitation, 12, 4. https://www.ncbi.nlm.nih.gov/pubmed/25576354 http://dx.doi.org/10.1186/1743-0003-12-4

Wang, X., Dong, W., Qiu, M., Ren, Z., Che, L., Dai, D., Wang, X., Yang, X., Sun, Z., Fu, B., Lee, S. Y., Xu, X., \& Zhang, D. H. (2008). HF(v' = 3) Forward Scattering in the F + H2 Reaction: Shape Resonance and Slow-Down Mechanism. Proceedings of the National Academy of Sciences of the United States of America, 105, 6227-6231.

https://www.ncbi.nlm.nih.gov/pubmed/18434547

http://dx.doi.org/10.1073/pnas.0710840105

Wu, T., Kansaku, K., \& Hallett, M. (2004). How Self-Initiated Memorized Movements Become Automatic: A Functional MRI Study. Journal of Neurophysiology, 91, 1690-1698. https://www.ncbi.nlm.nih.gov/pubmed/14645385 http://dx.doi.org/10.1152/jn.01052.2003

Wu, T., Chan, P., \& Hallett, M. (2008). Modifications of the Interactions in the Motor Networks When a Movement Becomes Automatic. Journal of Physiology, 586, 4295-4304. https://www.ncbi.nlm.nih.gov/pubmed/18617569 
http://dx.doi.org/10.1113/jphysiol.2008.153445

Yang, J. C., \& Chang, K. C. (2003). Exercise-Induced Acute Spinal Subdural Hematoma: A Case Report. Kaohsiung Journal of Medical Sciences, 19, 624-627.

https://www.ncbi.nlm.nih.gov/pubmed/14719560

http://dx.doi.org/10.1016/S1607-551X(09)70516-9

Zaidi, Z. H. (1959). Hula-Hoop Syndrome. Canadian Medical Association Journal, 80, 715-716. https://www.ncbi.nlm.nih.gov/pubmed/13652012

Zamor, C. (2009). Hooping: A Revolutionary Fitness Program. Health Journal, 45-48. http://www.lucashooping.com/files/Health_Magazine_Hooping_Health_reasons_Prevention _Aug_09.pdf

Submit or recommend next manuscript to SCIRP and we will provide best service for you:

Accepting pre-submission inquiries through Email, Facebook, LinkedIn, Twitter, etc. A wide selection of journals (inclusive of 9 subjects, more than 200 journals)

Providing 24-hour high-quality service

User-friendly online submission system

Fair and swift peer-review system

Efficient typesetting and proofreading procedure

Display of the result of downloads and visits, as well as the number of cited articles

Maximum dissemination of your research work

Submit your manuscript at: http://papersubmission.scirp.org/

Or contact psych@scirp.org 